\title{
ISO spectroscopy of star formation and active nuclei in the luminous infrared galaxy NGC 6240^
}

\author{
D. Lutz ${ }^{1}$, E. Sturm ${ }^{1}$, R. Genzel ${ }^{1}$, H. W. W. Spoon ${ }^{2}$, A. F. M. Moorwood ${ }^{3}$, H. Netzer ${ }^{4}$, and A. Sternberg ${ }^{4}$ \\ 1 Max-Planck-Institut für extraterrestrische Physik, Postfach 1312, 85741 Garching, Germany \\ e-mail: lutz@mpe.mpg.de; sturm@mpe.mpg.de; genzel@mpe.mpg.de \\ 2 Kapteyn Institute, PO Box 800, 9700 AV Groningen, The Netherlands \\ e-mail: spoon@astro.rug.nl \\ 3 European Southern Observatory, Karl-Schwarzschild-Str. 2, 85748 Garching, Germany \\ e-mail: amoor@eso.org \\ ${ }^{4}$ School of Physics and Astronomy and Wise Observatory, Raymond and Beverly Sackler Faculty of Exact Sciences, \\ Tel Aviv University, Ramat Aviv, Tel Aviv 69978, Israel \\ e-mail: netzer@wise1.tau.ac.il; amiel@wise1.tau.ac.il
}

Received 23 April 2003 / Accepted 22 July 2003

\begin{abstract}
We present Infrared Space Observatory mid- and far-infrared spectroscopy of the merging galaxy NGC 6240, an object presenting many aspects of importance for the role of star formation and AGN activity in [ultra]luminous infrared galaxies. The mid-infrared spectrum shows starburst indicators in the form of low excitation fine-structure line emission and aromatic "PAH" features. A strong high excitation [O IV] line is observed which most likely originates in the Narrow Line Region of an optically obscured AGN. NGC 6240 shows extremely powerful emission in the pure rotational lines of molecular hydrogen. We argue that this emission is mainly due to shocks in its turbulent central gas component and its starburst superwind. The total shock cooling in infrared emission lines accounts for $\sim 0.6 \%$ of the bolometric luminosity, mainly through rotational $\mathrm{H}_{2}$ emission and the [OI] $63 \mu \mathrm{m}$ line. We analyse several ways of estimating the luminosities of the starburst and the AGN in NGC 6240 and suggest that the contributions to its bolometric luminosity are most likely in the range 50-75\% starburst and $25-50 \%$ AGN.
\end{abstract}

Key words. galaxies: individual: NGC 6240 - infrared: galaxies - galaxies: active - galaxies: starburst

\section{Introduction}

The double nucleus merging galaxy NGC 6240 is a relatively nearby $\left(c z=7339 \mathrm{~km} \mathrm{~s}^{-1}, d=98 \mathrm{Mpc}\right)$ infrared-luminous system. At $L_{\mathrm{IR}}=6.05 \times 10^{11} L_{\odot}($ see e.g. Sanders \& Mirabel 1996 for the definition of the $8-1000 \mu \mathrm{m}$ luminosity $L_{\mathrm{IR}}$ ), it is almost an ultraluminous infrared galaxy and thus allows detailed studies of many aspects of the phenomenon of [ultra]luminous infrared galaxies ([U]LIRGs). For the dusty system NGC 6240, the infrared luminosity is identical to the bolometric luminosity to good approximation, the optical/near-infrared and Xray regions observed to be minor contributors. In the infrared selected sample studied spectroscopically with the Infrared Space Observatory ISO by Genzel et al. (1998) it is among the sources with indications for an active galactic nucleus (AGN) in the mid-infrared emission line spectrum, similar indications

Send offprint requests to: D. Lutz, e-mail: lutz@mpe.mpg.de

^ Based on observations with ISO, an ESA project with instruments funded by ESA member states (especially the PI countries: France, Germany, the Netherlands, and the UK) with the participation of ISAS and NASA. can be obtained from the low resolution mid-infrared diagnostic diagram of Laurent et al. (2000). Several X-ray studies have firmly established the presence of powerful AGN activity (e.g., Iwasawa \& Comastri 1998; Vignati et al. 1999), in fact occuring in both nuclei (Komossa et al. 2003). Coexistence of star formation and AGN activity is a feature of many [ultra]luminous infrared galaxies. The proximity of NGC 6240 and the extensive set of multi-wavelength observations make it a unique target for studying the quantitative contributions of the AGN and star formation activity in a [U]LIRG with a relatively powerful AGN. Such studies are not only of interest for an understanding of the local [U]LIRG population, but also for the high redshift infrared and submm selected populations to which the [U]LIRGs likely form the closest available local analogs.

A second important question relates to the evolution of merging galaxies like NGC 6240. Recent near-infrared dynamical studies (Genzel et al. 2001; Tacconi et al. 2002) suggest that merging ultraluminous infrared galaxies as a class will evolve into moderate mass, disky-type elliptical galaxies. NGC 6240 stands out in this group by its very high stellar 
Table 1. Log of the ISO observations of NGC 6240 presented in this paper. The number of wavelength ranges includes "serendipitous" data obtained by a different detector in different order simultaneously with a requested range, but excludes data outside the nominal SWS wavelength bands.

\begin{tabular}{llllcc}
\hline \hline Obs_ID & $\begin{array}{l}\text { Date } \\
\text { UT }\end{array}$ & AOT & Proposal & $N_{\text {Ranges }}$ & $\begin{array}{c}\text { Duration } \\
\text { h:mm:ss }\end{array}$ \\
\hline 09700106 & 22-Feb.-1996 & S02 & RGENZEL_MPEXGAL2 & 16 & $5: 06: 22$ \\
09700207 & 22-Feb.-1996 & S02 & RGENZEL_MPEXGAL2 & 11 & $2: 20: 14$ \\
31101401 & 23-Sep.-1996 & S02 & ASTERNBE_FEGALAXI & 2 & $0: 56: 40$ \\
31801036 & 29-Sep.-1996 & S02 & RGENZEL_MPEXGAL3 & 7 & $2: 53: 52$ \\
48101201 & 11-Mar.-1997 & S02 & PVDWERF_SHOCKS & 7 & $1: 49: 02$ \\
83300305 & 25-Feb.-1998 & S02 & EEGAMI_H2 & 3 & $1: 15: 26$ \\
83500308 & 27-Feb.-1998 & S02 & EEGAMI_H2 & 3 & $1: 00: 34$ \\
84400107 & 08-Mar.-1998 & S02 & EEGAMI_H2 & 8 & $2: 06: 38$ \\
86600118 & 30-Mar.-1998 & S02 & DLEVINE_FILLFUP & 3 & $0: 42: 02$ \\
27801108 & 21-Aug.-1996 & L01 & HSMITH_IRBGALS & n.a. & $1: 09: 04$ \\
81000771 & 02-Feb.-1998 & L02 & HSMITH_IRBGALS & n.a. & $0: 20: 38$ \\
81000872 & 02-Feb.-1998 & L02 & HSMITH_IRBGALS & n.a. & $0: 20: 38$ \\
81000973 & 02-Feb.-1998 & L02 & HSMITH_IRBGALS & n.a. & $0: 20: 38$ \\
81001074 & 02-Feb.-1998 & L02 & HSMITH_IRBGALS & n.a. & $0: 20: 38$ \\
81600475 & 08-Feb.-1998 & L02 & HSMITH_IRBGALS & n.a. & $0: 20: 40$ \\
81600676 & 08-Feb.-1998 & L02 & HSMITH_IRBGALS & n.a. & $0: 20: 40$ \\
\hline
\end{tabular}

velocity dispersion (Lester \& Gaffney 1994; Doyon et al. 1994) suggesting a very massive object. Spatially resolved dynamical studies using CO (cold gas; Tacconi et al. 1999), $\mathrm{H}_{2}$ (hot gas; van der Werf et al. 1993; Tecza et al. 2000), and stellar absorption features (Tecza et al. 2000) reveal complex, not fully relaxed dynamics with maximum velocity dispersion between the two nuclei. The extraordinary luminosity of NGC 6240 in the near-infrared rovibrational lines of molecular hydrogen (Joseph et al. 1984) may be partly linked to this complex dynamics, as well as to shocked emission in the superwind flow that is driven by the NGC 6240 starburst (van der Werf et al. 1993).

This paper presents ISO mid- and far-infrared spectroscopic observations of NGC 6240 and discusses implications for its starburst and AGN activity, and the nature of the powerful $\mathrm{H}_{2}$ emission.

\section{Observations and reduction}

We have retrieved from the ISO archive all observations of NGC 6240 that were obtained with the ISO Short Wavelength Spectrometer SWS (de Graauw et al. 1996). Table 1 lists the basic data. All observations were obtained in the SWS02 mode which produces short spectral segments at the nominal SWS resolving power ( 2000), centered on selected wavelengths. The data were reduced in the OSIA ${ }^{1} 3.0$ software framework which adopts the SWS calibration files of the offline processing software version 10.1. Standard reduction procedures were used, putting particular emphasis on identifying and eliminating individual noisy detectors and scans affected by signal jumps. Repeated observations of the same range were offset by small values to a consistent median flux. Single valued spectra were then produced by kappa-sigma clipping and averaging the many independent measurements inside a resolution element.

\footnotetext{
${ }^{1}$ OSIA is a joint development of the SWS consortium. Contributing institutes are SRON, MPE, KUL and the ESA Astrophysics Division.
}

A resolution element of $\lambda / 2000$ was used for this step. While leading to a slight degradation of the nominal SWS resolution, this is favourable for signal-to-noise reasons and does not affect the wide line profiles of NGC 6240 noticeably. Figure 1 shows the segments containing the detected lines and some ranges providing important upper limits. The spectra were rebinned with $R \sim 2000$ and are five times oversampled with respect to that resolution. Table 2 lists the derived fluxes (estimated to be accurate to $20-30 \%$ ), limits, and line parameters.

All observations were centered on the region of the NGC 6240 double nucleus (Fried \& Schulz 1983). The SWS apertures (between $14^{\prime \prime} \times 20^{\prime \prime}$ and $20^{\prime \prime} \times 33^{\prime \prime}$ ) include both nuclei as well as the extended region of emission in the near-infrared rovibrational lines of molecular hydrogen (e.g. van der Werf et al. 1993). Partial analyses of the SWS spectra of NGC 6240 using only a subset of the data with earlier calibrations and addressing only certain aspects are presented in Genzel et al. (1998) and Egami (1998; 1999).

We have also reduced an ISO-LWS 43-196 $\mu \mathrm{m}$ full spectrum (L01 mode) as well as 6 grating line scan observations (L02 mode) of the [N III] $57 \mu \mathrm{m}$ line (see Table 1). The data were first reduced in $\mathrm{LIA}^{2}$ version 8.1 , based on offline processing software version 10.1 calibration files. In particular dark current subtraction and absolute responsivity correction were done interactively. Following the LWS recipes for faint sources we have further reduced the data using ISAP $^{3}$ version 2.1. We deleted glitches and memory tails, and averaged multiple scans using ISAP's standard clip \& mean option with bin sizes preserving the sample spacing. To produce the continuous full grating spectrum

\footnotetext{
${ }^{2}$ LIA is a joint development of the ISO-LWS Instrument Team at Rutherford Appleton Laboratories (RAL, UK - the PI Institute) and the Infrared Processing and Analysis Center (IPAC/Caltech, USA).

3 The ISO Spectral Analysis Package (ISAP) is a joint development by the LWS and SWS Instrument Teams and Data Centers. Contributing institutes are CESR, IAS, IPAC, MPE, RAL and SRON.
} 
D. Lutz et al:: Starburst and AGN in the infrared spectrum of NGC 6240
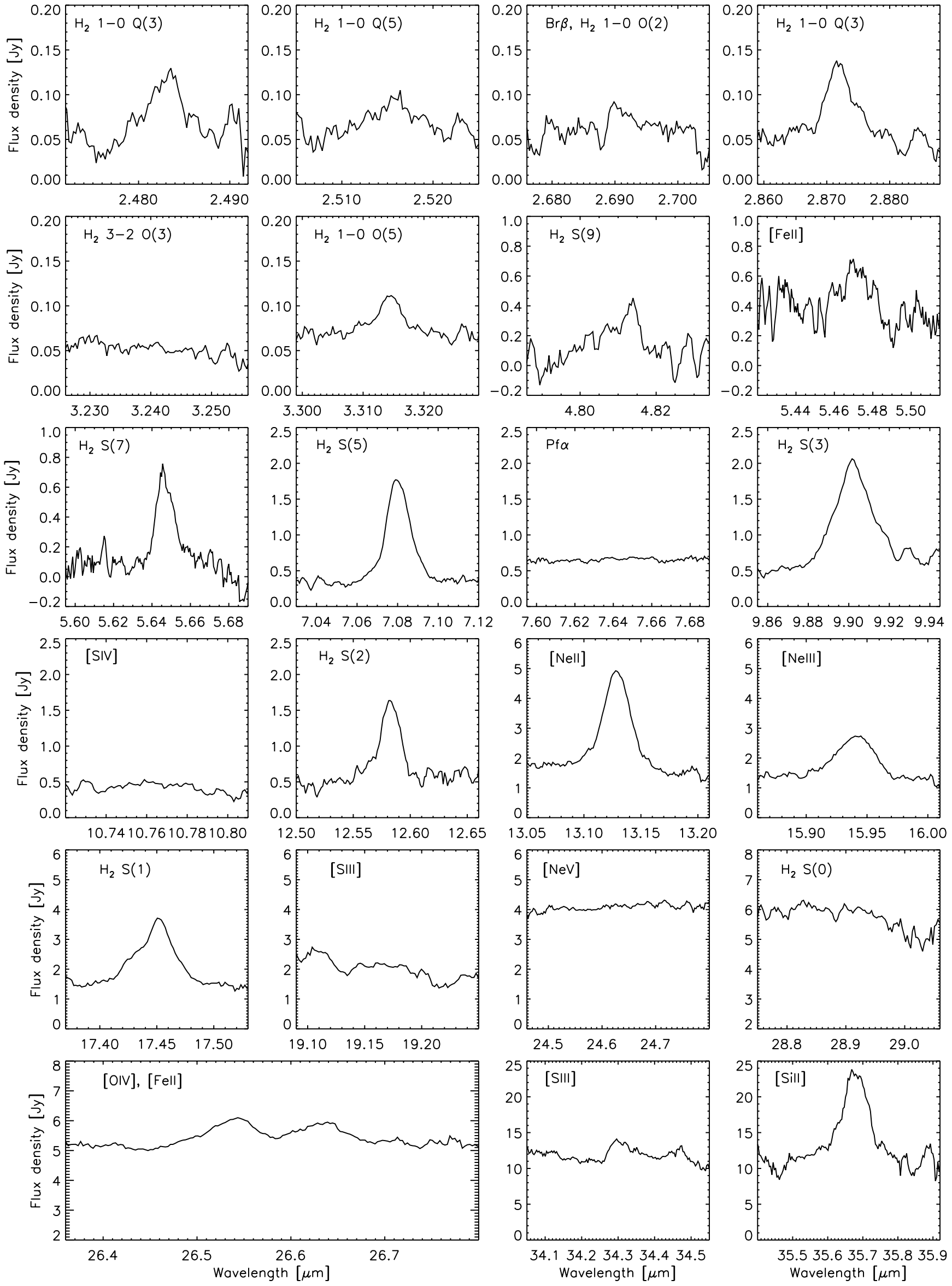

Fig. 1. ISO-SWS spectra of the lines detected in NGC 6240. Some of the ranges from which important upper limits are derived are also shown. 
Table 2. Line fluxes and limits measured with SWS and LWS for NGC6240. Upper limits for line fluxes are for an adopted FWHM of $600 \mathrm{~km} \mathrm{~s}^{-1}$ (SWS) which corresponds to an unresolved line for LWS. ${ }^{1}$ FWHM enforced for the Gaussian fit used to derive the line flux.

\begin{tabular}{|c|c|c|c|c|}
\hline Transition & $\begin{array}{c}\lambda_{\text {Rest }} \\
\mu \mathrm{m}\end{array}$ & $\begin{array}{r}\lambda_{\mathrm{Obs}} \\
\mu \mathrm{m}\end{array}$ & $\begin{array}{r}\text { Flux } \\
10^{-20} \mathrm{~W} \mathrm{~cm}^{-2} \\
\end{array}$ & $\begin{array}{r}F W H M \\
\mathrm{~km} \mathrm{~s}^{-1}\end{array}$ \\
\hline $\mathrm{H}_{2} 1-0 \mathrm{Q}(3)$ & 2.4237 & 2.4832 & 1.55 & 540 \\
\hline $\mathrm{H}_{2} 1-0 \mathrm{Q}(5)$ & 2.4548 & 2.5159 & 0.83 & 604 \\
\hline $\mathrm{H} \mathrm{Br} \beta$ & 2.6259 & & $<0.90$ & \\
\hline $\mathrm{H}_{2} 1-0 \mathrm{O}(3)$ & 2.8025 & 2.8720 & 1.59 & 548 \\
\hline $\mathrm{H}_{2} 2-1 \mathrm{O}(3)$ & 2.9741 & & $<0.35$ & \\
\hline $\mathrm{H}_{2} 3-2 \mathrm{O}(3)$ & 3.1638 & & $<0.30$ & \\
\hline $\mathrm{H}_{2} 1-0 \mathrm{O}(5)$ & 3.2350 & 3.3144 & 0.56 & 436 \\
\hline $\mathrm{H} \mathrm{Br} \alpha$ & 4.0523 & & $<2.5$ & \\
\hline $\mathrm{H}_{2} 0-0 \mathrm{~S}(11)$ & 4.1813 & & $<2.6$ & \\
\hline $\mathrm{H}_{3}^{+} 1,2,3_{+1} \rightarrow 3,3$ & 4.350 & & $<1.9$ & \\
\hline $\mathrm{H}_{2} \mathrm{O}-0 \mathrm{~S}(9)$ & 4.6946 & 4.8114 & 3.90 & $600^{1}$ \\
\hline$[\mathrm{Fe} \mathrm{II}]$ & 5.3402 & 5.4707 & 5.46 & 875 \\
\hline $\mathrm{H}_{2} 0-0 \mathrm{~S}(7)$ & 5.5112 & 5.6469 & 7.27 & 636 \\
\hline $\mathrm{H}_{2} 0-0 \mathrm{~S}(5)$ & 6.9095 & 7.0803 & 12.20 & 580 \\
\hline $\operatorname{HPf} \alpha$ & 7.4599 & & $<0.6$ & \\
\hline $\mathrm{H}_{2} 0-0 \mathrm{~S}(3)$ & 9.6649 & 9.9021 & 9.24 & 641 \\
\hline$[\mathrm{S} \mathrm{IV}]$ & 10.5105 & & $<1.1$ & \\
\hline $\mathrm{H}_{2} 0-0 \mathrm{~S}(2)$ & 12.2786 & 12.5821 & 4.35 & 484 \\
\hline$[\mathrm{Ne} I I]$ & 12.8136 & 13.1288 & 16.20 & 630 \\
\hline$[\mathrm{Ne} \mathrm{V}]$ & 14.3217 & & $<0.8$ & \\
\hline$[\mathrm{Ne}$ III $]$ & 15.5551 & 15.9405 & 6.31 & 675 \\
\hline $\mathrm{H}_{3}^{+} 5,0 \rightarrow 4,3$ & 16.331 & & $<1.0$ & \\
\hline $\mathrm{H}_{2} 0-0 \mathrm{~S}(1)$ & 17.0348 & 17.4497 & 7.83 & 657 \\
\hline$[\mathrm{Fe} \mathrm{II}]$ & 17.9360 & & $<1.6$ & \\
\hline [S III] & 18.7130 & & $<2.0$ & \\
\hline$[\mathrm{Ne} \mathrm{V}]$ & 24.3175 & & $<0.6$ & \\
\hline$[\mathrm{S} \mathrm{I}]$ & 25.2490 & & $<1.2$ & \\
\hline [O IV] & 25.8903 & 26.5384 & 2.33 & 672 \\
\hline$[\mathrm{Fe} \mathrm{II}]$ & 25.9883 & 26.6322 & 1.85 & 653 \\
\hline $\mathrm{H}_{2} 0-0 \mathrm{~S}(0)$ & 28.2188 & & $<2.2$ & \\
\hline [S III $]$ & 33.4810 & 34.3091 & 3.57 & 503 \\
\hline $\mathrm{OH} 1 / 2-3 / 2$ & 34.6164 & & $\tau<0.5$ & no absorption \\
\hline [Si II] & 34.8152 & 35.6786 & 26.0 & 731 \\
\hline$[\mathrm{Fe} \mathrm{II}]$ & 35.3487 & & $<2.4$ & \\
\hline$[\mathrm{Ne} \mathrm{III}]$ & 36.0135 & & $<2.6$ & \\
\hline $\mathrm{H}_{2} \mathrm{O} 6_{43} \rightarrow 5_{32}$ & 40.3367 & & $<3.7$ & \\
\hline [O III $]$ & 51.8145 & & $<14.0$ & \\
\hline [N III] & 57.3170 & & $<4.0$ & \\
\hline [OI] & 63.1837 & 64.744 & 72.00 & unresolved \\
\hline [O III] & 88.3560 & & $<6.0$ & \\
\hline$[\mathrm{N} \mathrm{II}]$ & 121.8976 & 124.95 & 2.3 & unresolved \\
\hline $\mathrm{H}_{2} \mathrm{O}_{13} \rightarrow 2_{02}$ & 138.5272 & & $<1.7$ & \\
\hline$[\mathrm{OI}]$ & 145.5254 & 148.99 & 4.00 & unresolved \\
\hline CO 17-16 & 153.2667 & & $<1.8$ & \\
\hline$[\mathrm{C} \mathrm{II}]$ & 157.7409 & 161.598 & 29.00 & unresolved \\
\hline
\end{tabular}

shown in Fig. 2 we have shifted the 10 LWS01 sub-spectra in offset mode by values between 5 and 20 percent $(30 \%$ for detector LW5), using detector LW04 ( $\approx 140$ to $170 \mu \mathrm{m})$ as reference ("good") detector. With an average effective LWS beam size of 77" (Gry et al. 2002) all relevant regions of NGC 6240 are covered. Fluxes or upper limits are included in Table 2. All measured lines are unresolved at the resolution of LWS grating spectra (150 to $300, \approx 1500 \mathrm{~km} \mathrm{~s}^{-1}$ ).
We have reanalysed the ISOPHOT-S spectrum already presented in Genzel et al. (1998) and Rigopoulou et al. (1999) using PIA ${ }^{4}$ version 9.0.1. The spectrum was obtained on 1996 February 15 using rectangular chopped mode (chopper throw of $180^{\prime \prime}$ ). The pure on-source integration time was $512 \mathrm{~s}$. Steps in the data reduction included: 1) deglitching on ramp level; 2) subdivision of ramps in four sections of 32 non destructive

${ }^{4}$ PIA is a joint development by the ESA Astrophysics division and the ISO-PHT consortium. 


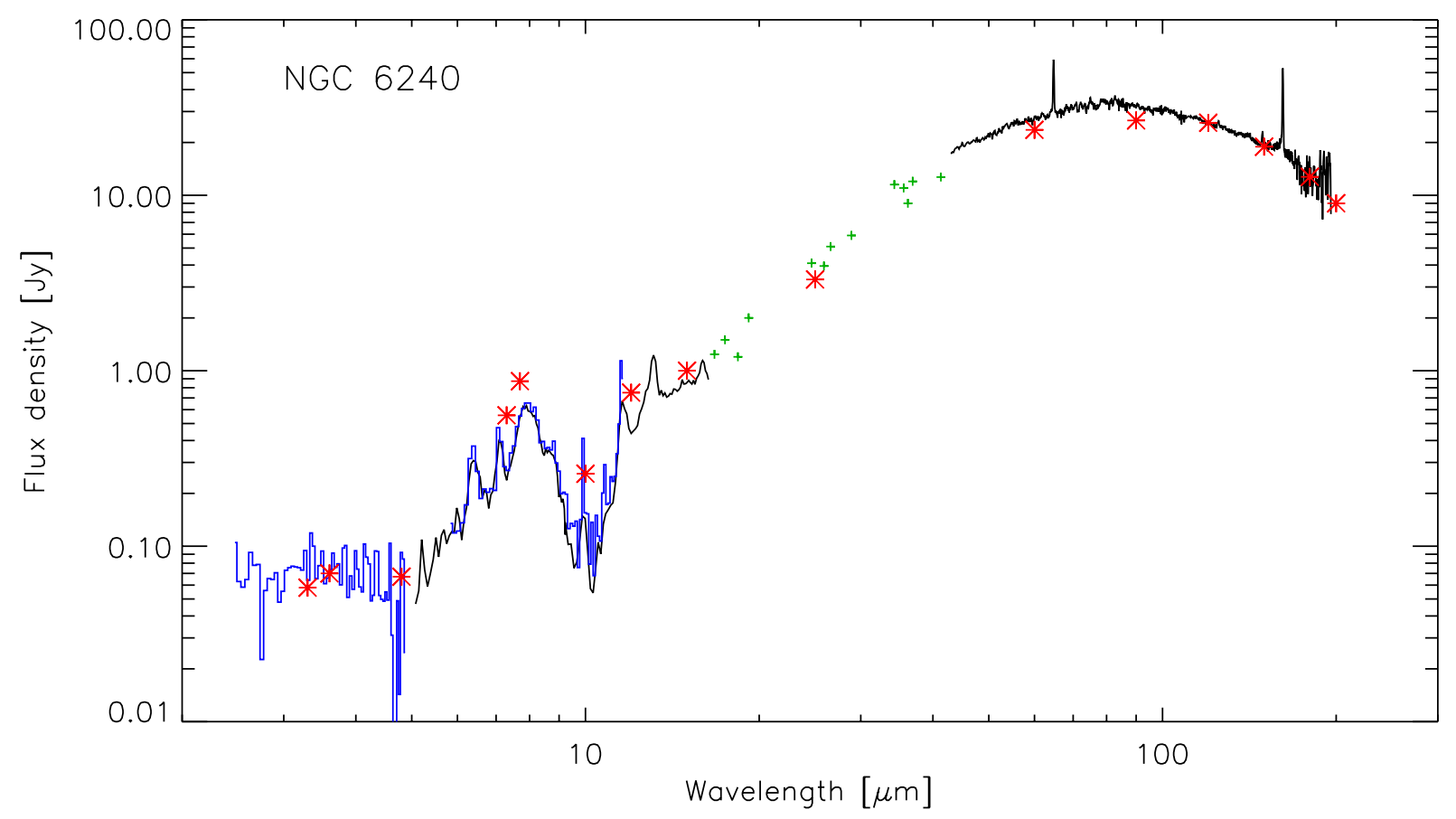

Fig. 2. Infrared spectral energy distribution of NGC 6240 from ISO observations. Continuous lines show the ISOCAM-CVF and LWS spectra, histograms the ISOPHOT-S spectrum. Small crosses indicate continua from the SWS spectra at $>16 \mu \mathrm{m}$, where the relative zodiacal light contribution is small. Asterisks indicate the broad band photometry of Klaas et al. (1997, 2001).

read-outs; 3) ramp fitting to derive signals; 4) masking of bad signals by eye-inspection; 5) kappa sigma and min/max clipping on remaining signal distribution; 6) determination of average signal per chopper plateau; 7) masking or correction of bad plateaux by eye-inspection; 8) background subtraction using all but the first four plateaux; 9) finally, flux calibration, using the signal dependent spectral response function. The absolute calibration is accurate to within $20 \%$. Figure 2 shows the infrared spectral energy distribution of NGC 6240 combining the ISOPHOT-S spectrum, the ISOCAM-CVF data of Laurent et al. (2000 and priv. communication), the LWS data and published broadband photometry.

\section{Results}

The mid-infrared fine-structure emission line spectrum of NGC 6240 resembles the spectra of starbursting infrared galaxies (Thornley et al. 2000; Förster Schreiber et al. 2001; Verma et al. 2003) in showing strong [Ne II] $12.81 \mu \mathrm{m}$ emission and a moderate excitation as probed by the ratio [Ne III] $15.55 \mu \mathrm{m} /$ [Ne II] $12.81 \mu \mathrm{m}$. It differs from a typical starburst spectrum in the enormous strength of the rotational emission lines of warm molecular hydrogen, and in the fairly strong emission in [O IV] $25.89 \mu \mathrm{m}$, a line which is one of the strongest tracers of the mid-infrared spectra of AGN (Sturm et al. 2002), but very weak or absent in the spectra of starbursts (Lutz et al. 1998; Verma et al. 2003).

The overall spectral energy distribution and mid-infrared low resolution spectrum (Fig. 2) also resemble star forming galaxies, with prominent emission in the mid-infrared aromatic "PAH" features. The rotational lines of molecular hydrogen are so strong that some of them are easily detected in the low resolution spectra.

\subsection{Detection of AGN Narrow Line Region emission}

The detection of [O IV] at $\sim 15 \%$ of the [Ne II] flux is clearly above the regime of starbursts and approaches values for AGN (Fig. 3). Under the assumption of simple mixing of AGN and starburst emission, the AGN will then contribute little to [Ne II] but will dominate [OIV]. A word of caution is in place here about possible other origins of [OIV]. The very low level [OIV] emission seen in starbursts is not generally attributed to AGN but to either supernova or wind-related ionizing shocks or very hot stars (Lutz et al. 1998; Schaerer \& Stasinska 1999). Given the dynamics and the superwind activity of NGC 6240 and the extremely strong and likely shock-related molecular hydrogen emission, it is conceivable that, compared to a normal starburst, ionizing shocks producing [OIV] are at a significantly elevated level as well. One way to probe for such a contribution is to look for other emissions that are strong in ionizing shocks. An obvious choice here is [Fe II] which is amply emitted in partially ionized zones of shocks (e.g. Graham et al. 1987; Hollenbach \& McKee 1989) and additionally boosted by shock destruction of grains (e.g. Jones et al. 1996; Oliva et al. 1999a,b) onto which Fe is normally depleted. Indeed both starbursts and supernova shocks observed with ISO show strong emission in the [Fe II] $25.98 \mu \mathrm{m}$ line that is neighbouring [OIV]. Similar ratios of the two lines are observed in starbursts and SNRs (Fig. 3). In AGN, however, the [Fe II] emission from shocked or UV/X-ray irradiated partially ionized zones is (relatively) fainter compared to the strong [OIV] emission from the NLR. The [Fe II] emission in NGC 6240 is fainter relative to [OIV] than in starbursts or supernova remnants which suggests the AGN to dominate [OIV]. This is quantified in the simple mixing model shown in Fig. 3 which assumes the 


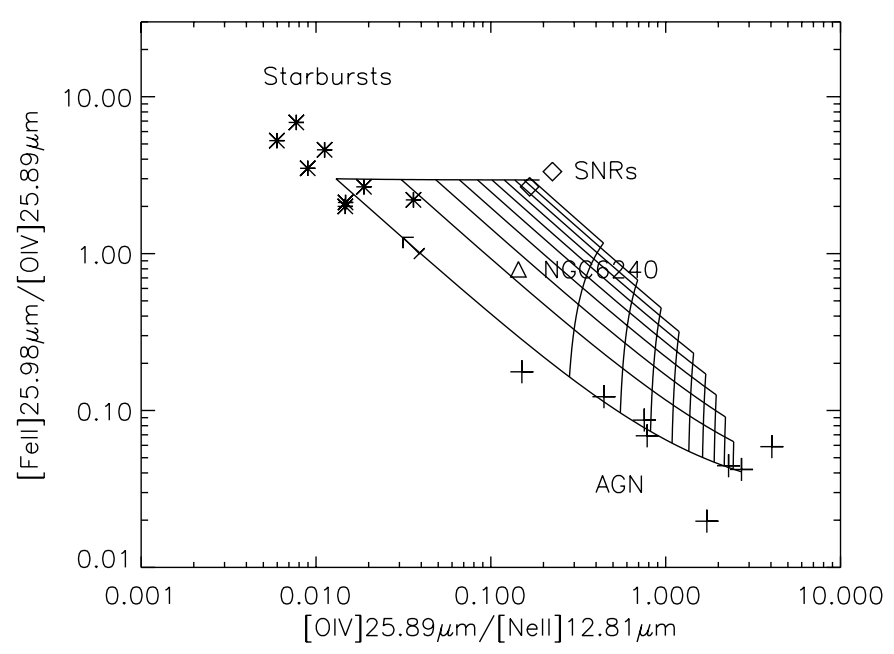

Fig. 3. Fine-structure emission line ratios for NGC 6240, compared to a number of bright AGN from Sturm et al. (2002), the starbursts of Verma et al. (2003, excluding low metallicity dwarfs), and the supernova remnants IC 443 and RCW 103 (Oliva et al. 1999a,b). The overplotted grid represents simple mixing by a linear superposition of starburst, SNR, and AGN line spectra. The loci of the grid lines indicate where the contribution to $[\mathrm{Ne} \mathrm{II}]$ is $0 \%, 10 \%, 20 \%, \ldots$ for supernova remnants and for AGN. The percentage contribution to other lines will be different. Note that the four leftmost AGN themselves have evidence (PAH emission) for significant star formation inside the SWS beam.

spectrum to be a linear superposition of a starburst, an AGN, and a SNR spectrum. At the location of NGC 6240, the contributions to [Ne II] are then about $82 \%$ starburst, $14 \%$ SNR and $4 \%$ AGN. Because of the strongly different intrinsic $[\mathrm{O} I \mathrm{~V}] /[\mathrm{Ne} \mathrm{II}]$ ratios, the contributions to [O IV] are very different: $7 \%$ starburst, $18 \%$ SNR, and a dominant $75 \%$ AGN. This nominal mix as well as the complexity of the excitation mechanisms for these lines clearly allow some of the strong [O IV] emission to be due to a high level of non-AGN shock activity, but we will proceed in the following with the assumption that [O IV] is mainly tracing the AGN proper.

The higher ionization potential NLR lines or coronal lines are weak or absent in NGC 6240. No near-infrared coronal line detections have been reported in the literature. This could be due to extinction. A limit of $<0.26$ can be derived for the extinction-insensitive ratio of [Ne V] $24.3 \mu \mathrm{m}$ and the neighbouring [OIV] $25.89 \mu \mathrm{m}$ line. This places the NLR of NGC 6240 towards the low end but within the excitation range of the Seyferts studied by Sturm et al. (2002), who measured ratios in the range from 0.18 to 0.37 . The data are consistent with [O IV] being AGN dominated.

Egami (1999) suggested an overall dominance of the AGN contribution to the NGC 6240 fine-structure line emission, not only for [O IV] but also for the low excitation lines like [Ne II]. To make this consistent with typical AGN NLR line ratios (e.g., Sturm et al. 2002), he invoked a model where the ionization structure of the NLR follows an onion-like layered pattern from high excitation inside to low excitation outside, with obscuration selectively blocking the higher excitation inner parts. High obscuration specifically towards part of the NLR is conceivable, also given the large amounts of gas in the central region of NGC 6240. We consider an extreme effect of obscuration on the line ratios unlikely, however, because of the non-ideal structure of real NLRs (e.g. Capetti et al. 1997) which does not show perfect layering of ionisation stages as in idealized spherically symmetric photoionisation models. In addition, such a scenario would either require the same peculiar geometry to apply for both AGN, or insignificance of one of them, which is in contrast to their modest hard X-ray flux ratio of $\sim 3$ (Komossa et al. 2003).

The combined evidence favours a model where the lower ionisation lines are dominated by starburst emission but [O IV] by the NLR(s) of the AGN(s). The absolute flux of [O IV] from the NLR may still be affected by obscuration. NLR obscuration cannot be constrained from the data that are lacking extinctionsensitive ratios which are purely tracing the NLR. In principle, the [O III] nondetections (Table 2) also might constrain properties of the obscured Narrow Line Region (e.g. [O III] $88 \mu \mathrm{m} /$ [O IV] $26 \mu \mathrm{m}<2.6)$. However, our ignorance about density and ionization parameter of this region prevents converting this into an obscuration constraint.

\subsection{Properties of the starburst}

At an excitation ratio [Ne III] $15.55 \mu \mathrm{m} /[\mathrm{Ne}$ II] $12.81 \mu \mathrm{m}$ of 0.39 , NGC 6240 is well within the range covered by normal and high metallicity starburst galaxies (Thornley et al. 2000; Verma et al. 2003). This is still true when lowering the ratio by correcting for the likely noticeable contamination of [Ne III] by AGN emission. Estimated from AGN [Ne III]/[O IV] ratios (Sturm et al. 2002) and the observed [O IV] flux, about half of the [Ne III] $15.55 \mu \mathrm{m}$ flux could be due to a relatively low excitation NLR, whereas AGN contamination of the stronger [Ne II] line is much less significant. A rough estimate of the intrinsic starburst neon excitation ratio is hence closer to 0.2 rather than the observed total of 0.39 . This is in line with the overall picture of an ageing starburst derived on the basis of near-infrared data, e.g. the low equivalent width of the nearinfrared hydrogen recombination lines (e.g., Tecza et al. 2000).

In the gas-rich circumnuclear region of NGC 6240, extinction is most likely significant. If possible, it should be estimated from mid-infrared tracers representative of the starburst activity probed by the ISO observations. The two most reliable estimators, recombination lines and the [S III] lines are of limited use for the NGC 6240 data, however. Because of their low equivalent width, no recombination lines are significantly detected in our data. Comparison of the [S III] $18.7 \mu \mathrm{m}$ line (inside the silicate feature) with the $33.48 \mu \mathrm{m}$ line can provide another estimate of obscuration which is effectively a lower limit based on the assumption of [S III] being in the low density limit. The $18.71 \mu \mathrm{m}$ line is indeed undetected, consistent with high obscuration, but the overall weakness of [S III] in NGC 6240 (as in other high-metallicity starbursts, see Verma et al. 2003) makes the limit on the ratio with [S III] $33.48 \mu \mathrm{m}$ still consistent with the intrinsic ratio, and thus prevents derivation of a lower limit on obscuration. Another way for obtaining an estimate for the obscuration is to compare the near-infrared Br $\gamma$ flux of Goldader et al. (1997), obtained in a $3^{\prime \prime} \times 9^{\prime \prime} \mathrm{N}-\mathrm{S}$ 
aperture, with the [Ne II] flux. The ratio of 0.012 is a factor of 2 lower than the one derived in matching apertures for $\operatorname{Br} \gamma$ and [Ne II] fluxes in M 82 (Förster-Schreiber et al. 2001). This suggests $10-15 A_{V}$ higher extinction towards NGC 6240 than the well-studied extinction towards M $82\left(A_{V} \approx 5\right.$ for the screen case, Förster-Schreiber et al. 2001), in total 15-20 $A_{V}$ in the simplifying screen assumption. This corresponds to $\sim 1 \mathrm{mag}$ extinction of [Ne II] and [Ne III]. This estimate is very uncertain for several reasons. It is sensitive to uncertainties in the flux of the weak $\operatorname{Br} \gamma$ line that is difficult to measure accurately on top of the stellar continuum. Adopting the smaller $\mathrm{Br} \gamma$ flux of $1.15 \times 10^{-21} \mathrm{~W} \mathrm{~cm}^{-2}$ obtained by integrating over a $5^{\prime \prime}$ diameter aperture in the $\mathrm{Br} \gamma$ map of Tecza et al. (2000) increases $A_{V}$ by another $\approx 9$ mag. The estimate also relies on the assumption of similar metallicities in NGC 6240 and M 82. Our obscuration estimate is consistent with an estimate from the beginning of this century of $N_{\mathrm{H}} \approx 2 \times 10^{22}\left(A_{V} \approx 10\right)$ for the atomic hydrogen column in front of the radio continuum sources (Beswick et al. 2001). Since this column refers only to atomic gas, it is effectively a lower limit to which any molecular column would have to be added.

The picture of a low excitation starburst at obscuration somewhat above or similar to that of M 82 is also consistent with the $4 \sigma$ detection of the low excitation [N II] $122 \mu \mathrm{m}$ line, at a wavelength much less sensitive to obscuration. The ratio of 0.14 to [Ne II] is slightly above the estimated ratio 0.12 in M 82 (Colbert et al. 1999 vs. aperture-corrected Förster-Schreiber et al. 2001). The small critical density of the [N II] line could additionally affect this ratio. The limits on the higher excitation lines above $50 \mu \mathrm{m}$ ([N III], [O III]) are close to fluxes expected for an M 82-like excitation; slightly deeper spectra should detect these lines.

\subsection{Extremely strong emission of warm molecular hydrogen}

Even more than the near-infrared rovibrational transitions, the mid-infrared rotational transitions of $\mathrm{H}_{2}$ are extremely strong in NGC 6240. The observed rotational lines in the range 0-0 S(0) to $\mathrm{S}(11)$ (Table 2) carry a total flux of $4.5 \times 10^{-19} \mathrm{~W} \mathrm{~cm}^{-2}$. Adding an approximate $1 / 3$ for the unobserved among the pure rotational transitions, this corresponds to a luminosity of the $\mathrm{H}_{2}$ rotational lines of $1.8 \times 10^{9} L_{\odot}$ which is $\approx 0.3 \%$ of the infrared $(8-1000 \mu \mathrm{m})$ luminosity of the galaxy. A convenient way to visualize the properties of the $\mathrm{H}_{2}$ emission is an excitation diagram (Fig. 4, adopting transition probabilities of Turner et al. 1977, see also Wolniewicz et al. 1998). Thermally excited $\mathrm{H}_{2}$ at a single temperature and high density will show all transitions on a straight line in such a diagram. The concave curvature seen in Fig. 4 indicates a mix of temperatures, similar to excitation diagrams for starbursts and AGN observed with ISO (Rigopoulou et al. 2002). The rotational emission in NGC 6240 is, however, not only scaled up but also shows a stronger population of the higher rotational levels. At the low energy end, both the limit $T>145 \mathrm{~K}$ obtained from the limit on the $\mathrm{S}(1) / \mathrm{S}(0)$ ratio and the excitation temperature of $\sim 365 \mathrm{~K}$ derived from the $S(1)$ and $S(2)$ lines are within the ranges

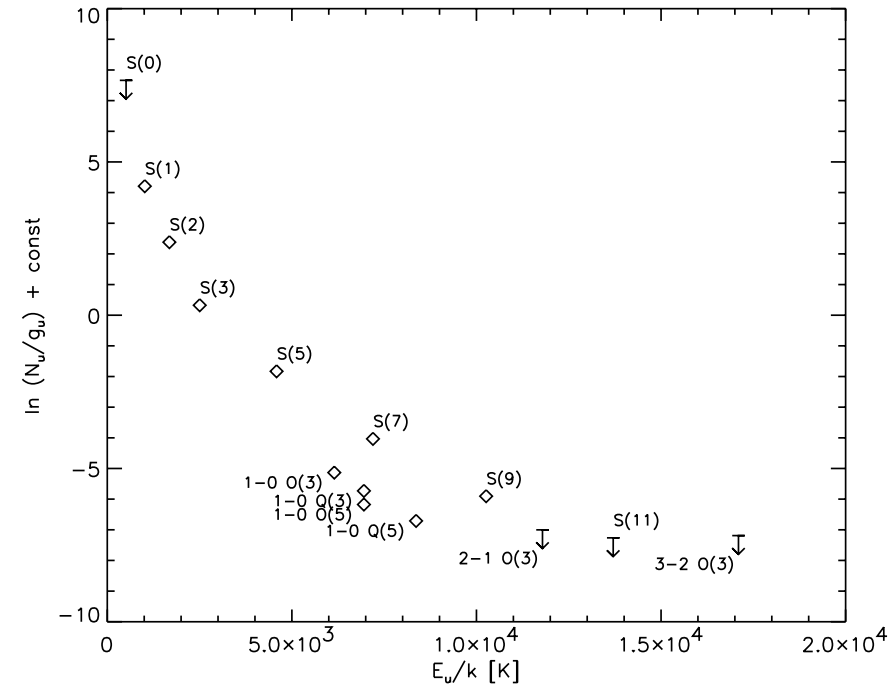

Fig. 4. $\mathrm{H}_{2}$ excitation diagram for NGC 6240, showing for the lines observed with SWS the upper level population divided by the level degeneracy, as a function of upper level energy. The level populations are derived from observed fluxes without extinction correction.

derived from those lines in other galaxies observed with ISO. Comparing lines originating at higher and lower energies, however, the flux ratio $S(5) / S(1)=1.56$ is larger than in any of the starbursts and AGN presented by Rigopoulou et al. (2002).

The upper level of the $S(3)$ line which falls near the center of the silicate feature appears somewhat underpopulated in Fig. 4, suggesting significant extinction of $A_{9.6} \mu \mathrm{m} \approx 1 \mathrm{mag}$ towards the $\mathrm{H}_{2}$ emitting region. The levels in the first vibrationally excited state from which the 1-0 lines arise appear underpopulated as well. Extinction may contribute here as well, since the lines from the first vibrationally excited state are at shorter wavelengths. For most commonly adopted extinction laws the obscuration at $2.4-3.2 \mu \mathrm{m}$ will be similar or less than the extinction at $9.6 \mu \mathrm{m}$, however. Less than or about one magnitude of extinction is not sufficient to explain the difference between the population of the vibrational ground state and first vibrational excited state. Subthermal population of the vibrational levels at not too high densities may play a role.

A total mass of $2-5 \times 10^{8} M_{\odot}$ for the "warm" (a few hundred K) molecular hydrogen can be estimated from the observed S(1) flux, and making the plausible assumption that the $S(1)$ emission originates in gas that on average is 50 $100 \mathrm{~K}$ colder than the excitation temperature estimated from $\mathrm{S}(2) / \mathrm{S}(1)$. This is a few percent of the total molecular gas mass estimated from CO observations (Solomon et al. 1997; Tacconi et al. 1999), a fraction not uncommon in starbursts and AGN. More significantly, $\approx 5 \times 10^{6} M_{\odot}$ of gas at an excitation temperature of $\sim 1100 \mathrm{~K}$ are needed to produce the observed $\mathrm{S}(5)$ flux.

Draine \& Woods (1990) proposed a model where most of the near-infrared rovibrational $\mathrm{H}_{2}$ emission of NGC 6240 is due to molecular gas illuminated by shock-produced soft $\mathrm{X}$-rays. Such gas would also contribute to a lesser degree to the observed rotational line emission. An observational test for this model is the expected emission of $\mathrm{H}_{3}^{+}$, e.g. at 4.35 and $16.33 \mu \mathrm{m}$. We do not detect these lines but the derived $3 \sigma$ limits (Table 2) are factors of 19 and 4 respectively above the 
predictions. They do not constrain the X-ray illumination scenario, also considering the much lower predictions of Maloney et al. (1996).

Radiative excitation of molecular hydrogen by UV illumination in moderate density PDRs and subsequent cascading leads to a strong population of the higher vibrationally excited levels which will be found above the pure rotational lines of similar energy in an excitation diagram (e.g., Fig. 3 of Timmermann et al. 1996; Sternberg \& Neufeld 1999, Fig. 6 of Draine \& Bertoldi 1999). The limits set on the 2-1 O(3) and 3-2 $\mathrm{O}(3)$ lines rule out a dominant contribution from such moderate density PDRs for the NGC $6240 \mathrm{H}_{2}$ spectrum. The higher vibrational levels are clearly not overpopulated with respect to the lower ones. This agrees with conclusions that have been reached on the basis of near-infrared spectra (e.g. van der Werf et al. 1993; Sugai et al. 1997).

Beyond excluding UV excitation in low density PDRs as the prime contributor, the thermal $\mathrm{H}_{2}$ spectrum observed in the SWS range holds little direct clue as to its sources of excitation. However, the detailed multiwavelength observations available for NGC 6240 make a strong contribution of shocks likely. The $\mathrm{CO}$ velocity field in the inner gas concentration is highly turbulent, suggesting rapid dissipation in this large gas mass (Tacconi et al. 1999). Shocks that will occur under such conditions are efficient sources of rotational $\mathrm{H}_{2}$ emission up to fairly high excitations, with the excitation diagram generally showing a smooth trend with level energy (e.g., OMC-1, Figs. 2 and 5 of Rosenthal et al. 2000). The [O I] $63 \mu \mathrm{m}$ line expected to be strong in shocks (Hollenbach \& McKee 1989) is also observed to be prominent in NGC 6240 (Table 2). Its flux is $\sim 2.5$ times that of [C II] $157 \mu \mathrm{m}$, unusually high compared to other starbursts and IR-bright galaxies (e.g., Fischer et al. 1999; Colbert et al. 1999). This high [O I]/[C II] ratio is even above the relatively high $[\mathrm{O} \mathrm{I}] /[\mathrm{C} \mathrm{II}]$ ratios observed in some [C II] deficient galaxies (Malhotra et al. 2001; Luhman et al. 2003) which are often ascribed to intensely heated (high $G_{0} / n$ ) PDRs. The very high ratio for NGC 6240 is probably not more than partially of the same origin - at $\log \left(L_{[\mathrm{CII}]} / L_{\mathrm{FIR}}\right)=-2.7$ (see also Stacey et al. 1991; Luhman et al. 1998) NGC 6240 is not [C II]-deficient. Morphology, kinematics and excitation conditions of the near-infrared rovibrational $\mathrm{H}_{2}$ lines have been variously used to argue for shock excitation of these lines as well (e.g., van der Werf 1993; Sugai et al. 1997). Single velocity/density C shock models (Kaufman \& Neufeld 1996) may not be able to reproduce simultaneously all the rotational lines. This can be plausibly accounted for by a mix of speeds and by a contribution of PDRs to the lowest rotational transitions.

Depending on shock conditions, cooling by the pure rotational lines of $\mathrm{H}_{2}$ will correspond to a few percent to tens of percent of the mechanical luminosity of the shock (e.g. Kaufman \& Neufeld 1996). The mechanical luminosity corresponding to the observed rotational $\mathrm{H}_{2}$ cooling may thus be approaching $10^{10} L_{\odot}$. A refined estimate is possible considering other potential major shock cooling lines. We do not detect in the LWS spectrum lines of $\mathrm{CO}$ and $\mathrm{H}_{2} \mathrm{O}$ that can also be major coolants for shocks in molecular regions. Table 2 includes limits for $\mathrm{CO}$ and $\mathrm{H}_{2} \mathrm{O}$ lines in the most sensitive regions of the spectrum. These are factors $\sim 5$ less than the fluxes of the strongest rotational molecular hydrogen lines. We may use these limits and the ratio $S(5)$ to $1-0 \mathrm{Q}(3)$ to search for acceptable conditions in the range of shock speeds and densities sampled by the shock models of Kaufman \& Neufeld (1996). These argue for, on average, relatively fast $\left(V \sim 30 \mathrm{~km} \mathrm{~s}^{-1}\right)$ shocks in gas of preshock density $\sim 10^{5} \mathrm{~cm}^{-3}$. Under these conditions, the rotational lines of molecular hydrogen will radiate $\sim 40 \%$ of the shock mechanical luminosity.

The strong and probably shock dominated [O I]63 $\mu \mathrm{m}$ line indicates that this picture is not complete. It radiates another $0.3 \%$ of the bolometric luminosity but is not produced in significant quantities in the nondissociative (C-) shock models cited above, where oxygen is assumed mostly locked in molecules (but cf. Poglitsch et al. 1996). Strong [O I] emission is however a key result of models of dissociative (J-) shocks (Hollenbach \& McKee 1989). A single shock model is not adequate to reproduce a galaxy with a wide range of shock speeds and preshock densities. Assuming that the $\mathrm{H}_{2}$ rotational lines and [OI] are the strongest coolants of shocks in NGC 6240, the total mechanical luminosity of these shocks must be of the order $10^{10} L_{\odot}$ even if some fraction of those lines has other origins.

If derived from the kinetic energy of the turbulent central gas concentration $\left(\sim 3 \times 10^{9} M_{\odot}, \sigma \approx 150 \mathrm{~km} \mathrm{~s}^{-1}\right.$, Tacconi et al. 1999), a shock mechanical luminosity of $10^{10} L_{\odot}$ will dissipate this turbulent energy within a few million years, i.e. on the dynamical timescale of the central gas concentration. Additional contributions may come from the mechanical luminosity of the starburst "superwind" in NGC 6240, estimated to be of the order $10^{10} L_{\odot}$ (e.g., Heckman et al. 1990; Schulz et al. 1998).

\section{What are the contributions of star formation and AGN to the luminosity of NGC 6240?}

We discuss several approaches to quantify the luminosity of the starburst and the AGN(s) in NGC 6240. All these approaches directly relate a mid-infrared or X-ray observable to the infrared (or bolometric) luminosity. In this they differ from the purely mid-infrared diagnostic diagrams (Genzel et al. 1998; Laurent et al. 2000) where extrapolation from mid-infrared to bolometric has to be considered separately (e.g. Sect. 3.6 of Genzel et al. 1998). The uncertainties will thus include the uncertainties of the extrapolation to bolometric luminosity. In addition to normal uncertainties in measurements and methods, a consistent difficulty of several of these approaches is incomplete characterisation of obscuration. Effectively, this leads to a situation where a lower limit to the luminosity of a component can be set with some confidence, but where it is very difficult to assess whether more of this component is present at higher obscuration or whether a different component takes over. It is then more useful to quantify what is observed, effectively defining a lower limit, than to make possibly biased interpretations of the obscured components. It is also worth remembering that obscuration towards spatially distinct regions (e.g., starburst, Narrow Line Region, AGN dust source, AGN X-ray source) is likely to differ considerably.

Since modelling from first principles is currently unreliable for several of the constraints discussed below, we follow an 
approach of quantitative but empirical comparison to starburst and AGN templates. For NGC 6240 as well as for the (dusty) comparison starbursts we assume the bolometric luminosity to be equal to the $8-1000 \mu \mathrm{m}$ infrared luminosity, while the relation between these quantities is explicitly included for AGN. For brevity, we do not include the radio emission of NGC 6240 in our discussion. The radio results summarized by Beswick et al. (2001) are consistent with coexistence of starburst and AGN, as outlined below.

\subsection{Star formation: Low excitation fine-structure lines}

With [Ne II] mainly excited by star formation, this line can be used to estimate the ionizing luminosity and bolometric luminosity of the starburst. During the evolution of a starburst, the ratio of ionizing luminosity of the hot stars to bolometric luminosity will decay, as will the excitation observed in the mid-infrared fine-structure lines (see, e.g., the models presented in Thornley et al. 2000). When trying to estimate the bolometric luminosity of the starburst component from the observed low excitation fine structure lines, it is hence important to choose templates with excitation similar to the ratio [Ne III] $15.55 \mu \mathrm{m} /[\mathrm{Ne}$ II] $12.81 \mu \mathrm{m} \approx 0.2$ derived above for the NGC 6240 starburst. Of the starbursts observed by Verma et al. (2003) this ratio is between 0.1 and 0.4 for M 82, NGC 3256, and NGC 3690/IC 694. For these galaxies, we compute a mean $\log \left(L_{[\mathrm{NeII}]} / L_{\mathrm{IR}}\right)=-3.03$. The values for the individual galaxies are between -2.89 and -3.22 . To meaningfully compare with the IRAS FSC-based infrared luminosities, we have considered their spatial extent compared to the SWS aperture by adding the two apertures for the two pointings on NGC 3690/IC 694 which together fully cover the active regions of this interacting system (see Fig. 1 in Verma et al. 2003) and by doubling the observed flux for M 82 where the SWS aperture only partially covers the starburst region (see Förster Schreiber et al. 2001).

Using this empirical calibration, the observed [Ne II] luminosity of $4.8 \times 10^{8} L_{\odot}$ converts into a starburst bolometric luminosity of $5 \times 10^{11} L_{\odot}$, i.e. the major part of the bolometric luminosity of NGC 6240. This would be an underestimate if the obscuration were higher than in the starburst templates. While there is evidence for higher obscuration of the NGC 6240 starburst than in M 82 (Sect. 3.2), we stick with the conservative assumption of similar obscuration because of the uncertainty of this estimate and of obscuration estimates for the other templates NGC 3256 and NGC 3690. The starburst luminosity would be overestimated if the metallicity were much higher than for the templates, which are already at supersolar metallicities, however (Verma et al. 2003). It would also be overestimated if the AGN contribution to [Ne III] were less, making the starburst more highly excited with a correspondingly lower ratio of total to ionizing luminosity.

\subsection{Star formation: Aromatic emission features}

The mid-infrared aromatic "PAH" emission features are observed over a wide range of interstellar medium conditions from the diffuse ISM to star forming regions but are not observed to be strong in the immediate vicinity of AGN. A number of studies have successfully decomposed low resolution mid-infrared spectra of galaxies into three components: A "PDR" component dominated by the aromatic emission features, an "H II" very small grain continuum steeply rising towards longer wavelengths and a hotter "AGN" dust continuum (Laurent et al. 2000; see also Sturm et al. 2000; Tran et al. 2001; Förster Schreiber et al. 2003). Dust obscuration can be important (Lutz et al. 1998a; Tran et al. 2001), with the additional complication of ice features (Spoon et al. 2002). The NGC 6240 spectrum (Fig. 5) is similar to starburst spectra which are a superposition of "PDR" and "H II", but there is an additional continuum extending down to $5 \mu \mathrm{m}$, indicative of an AGN continuum which we will discuss in Sect. 4.4. Subtracting such a continuum, the peak flux density in the $7.7 \mu \mathrm{m}$ aromatic feature is $\approx 0.45 \mathrm{Jy}$.

The physics and chemistry of the carriers of the aromatic features and their transient heating by UV photons originating over a very wide range of stellar types is complex and not fully understood. This certainly provides for a less direct link from PAH luminosity to the bolometric luminosity of a starburst than from luminosity of the emission lines originating in the medium ionized by OB stars to bolometric luminosity of the starburst. Nevertheless, it is worth trying to extrapolate from the PAH emission to the associated bolometric emission by using starbursts as templates. In order to minimize aperture effects in the comparison to the IRAS FSC-based infrared luminosity, we use objects for which PAH data are available covering a major part of the IRAS beam and/or the starburst region $^{5}$. Comparing the PAH $7.7 \mu \mathrm{m}$ feature peak flux density in $\mathrm{Jy}$, and the 8-1000 $\mu \mathrm{m}$ IR flux in $\mathrm{W} \mathrm{m}^{-2}$, we obtain a mean $\log \left(S_{7.7} / F_{\mathrm{IR}}\right)$ of 11.84 with a dispersion of 0.25 . Application of this relation to NGC 6240 results in a starburst luminosity of $2 \times 10^{11} L_{\odot}$.

This is an underestimate if the PAH emission is more obscured than in the comparison starbursts. Indications for the corresponding relative weakening of the 8.6 and $11.3 \mu \mathrm{m}$ features (in the wings of the silicate absorption) are not strong, however (Fig. 5). More important for assessing the robustness of this estimate are variations of the aromatic feature emission with environment. It is known since the IRAS mission that the aromatic features decrease when approaching a hot star or when going inwards from PDRs to H II regions (e.g., Boulanger et al. 1988; Verstraete et al. 1996). Correspondingly, trends are observed in "normal star forming" galaxies between the average radiation field intensity, echoed in the $60 / 100 \mu \mathrm{m}$ flux ratio tracing the large grain temperature, and the ratio of PAH emission to total infrared luminosity (Dale et al. 2001). This introduces additional uncertainties when extrapolating from PAH features to total luminosity and may cause underestimates if much of the star formation is concentrated in a small region.

\footnotetext{
5 ISOCAM-CVF M 82, M 83, NGC 253, NGC 520, NGC 1808, NGC 3690, NGC 4038/39, Arp 236, NGC 7252 (O. Laurent. priv. comm.) and NGC 3256 ISOPHOT-S (Rigopoulou et al. 1999).
} 

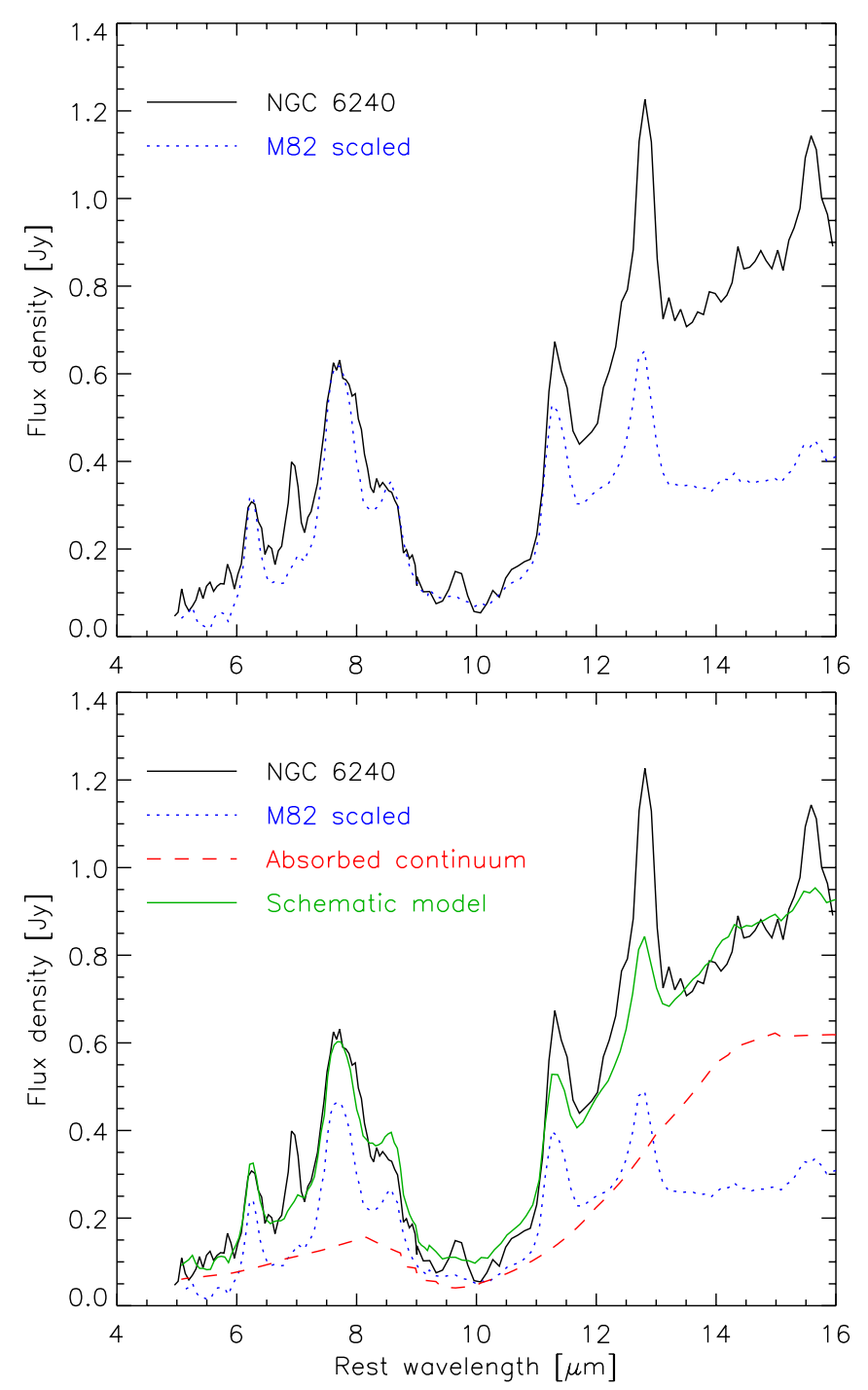

Fig. 5. Low resolution ISOCAM 5-16 $\mu \mathrm{m}$ spectrum of NGC 6240 compared to a scaled spectrum of M 82 (top) and a schematic model consisting of the sum of a scaled M 82 spectrum and an absorbed power law continuum (bottom). Obscuration of the continuum is needed for a satisfactory representation including the region of silicate feature. The schematic model is inadequate in the regions of strong emission lines because of differences in the emission line spectra of NGC 6240 and M 82.

\subsection{AGN: High excitation lines from the Narrow Line Region}

Here, we use the Narrow Line Region [O IV] $25.89 \mu \mathrm{m}$ luminosity as an indicator of the AGN luminosity and use again an empirical calibration. To avoid biasing our result by star formation contributions to the bolometric luminosity of comparison objects, we choose as comparison those among the Seyferts observed by Sturm et al. (2002) that have measured [O IV] lines and are not listed in their Table 1 as having strong evidence for circumnuclear star formation. The mean $\log \left(L_{[\mathrm{OIV}]} / L_{\mathrm{IR}}\right)$ of these 16 objects is -3.01 , with a dispersion of 0.29 . Using this calibration, the observed [O IV] luminosity of $7 \times 10^{7} L_{\odot}$ converts to an AGN infrared luminosity of $7 \times 10^{10} L_{\odot}$. This is an extrapolation based on the relation of [O IV] and infrared luminosities in the comparison Seyferts. In Seyfert galaxies, the $10-1000 \mu \mathrm{m}$ infrared range will represent very roughly $1 / 2$ of the bolometric luminosity (Spinoglio et al. 1995), with significant emission emerging at shorter wavelengths in the near-infrared, visual, and UV to X-ray ranges. The shorter wavelength emission in the integrated SEDs of Seyferts as in Spinoglio et al. (1995) will not always be pure AGN emission because of host contributions, but we assume it is dominated by the AGN. For an obscured AGN in NGC 6240, it is then plausible to assume that much of the NIR/optical/UV/soft-X part of the AGN bolometric luminosity would be absorbed and reradiated in the mid- and far-infrared. We hence double the estimate of the AGN contribution to the infrared luminosity to $1.4 \times 10^{11} L_{\odot}$.

This is an overestimate if the non-AGN shock contribution to [O IV] were large in NGC 6240, contrary to the estimate in Sect. 3.1. While we have tried to exclude objects with PAHs indicating strong star formation from the comparison sample, it may still be an overestimate if the IR emission of the comparison objects still has significant contributions from star formation. This is possible because of aperture effects between the PAH measurements and the FIR continuum measurements in currently available data. An example for this problem is NGC 1068 with very weak PAH in the ISOPHOT beam, and considerable star formation outside this beam but inside the IRAS beam (Telesco \& Decher 1988; Le Floc'h et al. 2001). Similar effects are hard to firmly exclude for other less well studied large galaxies because of the mismatch between the IRAS beam and the ISOPHOT-S aperture used to search for PAHs indicating star formation. High spatial resolution both in mid-infrared spectrophotometry and in the far-infrared would be needed to minimize these problems. Our AGN luminosity is an underestimate if the NLR of NGC 6240 is significantly obscured even at $26 \mu \mathrm{m}$. Several magnitudes of visual extinction needed to hide the NLR in the optical do not yet have a significant effect, however.

\subsection{AGN: Mid-Infrared continuum}

Mid-infrared spectra of AGN show a strong continuum due to warm dust in the vicinity of the AGN, either in the putative torus or on larger scales e.g. embedded in the Narrow Line Region. The spectrum of NGC 6240 has excess continuum over a pure starburst spectrum which may either be AGN related or an increased contribution of the steep "H II" continuum. The excess continuum must be obscured in order not to violate the observed spectrum in the region of the silicate feature. Because of this obscuration and the steep rise of a starburstrelated "H II" excess, an excess AGN continuum is best quantified shortwards of the PAH complex, at $\sim 5.9 \mu \mathrm{m}$ (Laurent et al. 2000). Subtracting from the observed spectrum of NGC 6240 a scaled M 82 spectrum reproducing the 6.2 and $7.7 \mu \mathrm{m}$ PAH features, we estimate the excess AGN continuum to be $\sim 0.07 \mathrm{Jy}$ at $5.9 \mu \mathrm{m}$ (Fig. 5).

Again we use AGN without evidence for circumnuclear star formation (no or only weak PAH features according to ISOPHOT-S observations) as templates. From 40 such AGN 
with $z<0.1$ and observations in the ISO archive we estimate a mean relation between $5.9 \mu \mathrm{m}$ continuum flux density in Jy, and the IR flux in $\mathrm{W} \mathrm{m}^{-2}$, of $\log \left(S_{5.9} / F_{\text {IR }}\right)=11.70$ with a dispersion of 0.29. Application of this relation to NGC 6240 results in an AGN infrared luminosity of $4.1 \times 10^{10} L_{\odot}$. Again, we have to consider that for the comparison Seyferts a noticeable part of the bolometric will emerge at short wavelengths that are likely absorbed and redistributed to the infrared in NGC 6240. As for the estimate based on the [O IV] line, we apply an additional factor 2 correction to account for the difference between infrared and bolometric luminosity in the comparison Seyferts, arriving at an AGN bolometric luminosity of $\approx 8 \times 10^{10} L_{\odot}$.

This is an underestimate if the AGN dust continuum in NGC 6240 is heavily obscured. Relatively little obscuration is sufficient to produce the silicate absorption needed for a spectrum fit (e.g. $A_{V} \approx 20$ in Fig. 5), but high values are not excluded. Another potential bias which can cause an underestimate of the AGN luminosity is due to the choice of $\mathrm{PAH}-$ free comparison objects. If unification related orientation effects cause variations in the AGN mid-IR brightness (Clavel et al. 2000), then our comparison objects selected to have a bright continuum standing out of the host galaxy PAHs might be above average in mid-IR brightness compared to an orientation-unbiased sample. In the absence of observations that can spatially separate host and AGN at mid and far-infrared wavelengths this bias is hard to break. Again, we will have overestimated the AGN luminosity if the IR luminosities of the comparison objects still contain significant starburst contributions, outside the ISOPHOT-S beam but inside the IRAS beam. We will also have overestimated the AGN luminosity if some of the excess $5.9 \mu \mathrm{m}$ continuum is still $\mathrm{H} \mathrm{II} \mathrm{re-}$ gion related.

\subsection{AGN: X-ray emission}

The X-ray emission of NGC 6240 holds important clues to the nature of its AGN. The soft X-ray emission (0.5-2 keV) is still dominated by extended thermal emission tracing the starburst superwind (Komossa et al. 1998; Iwasawa \& Comastri 1998). The hard X-rays (2-10 keV) are dominated by AGN emission reflected by cold and warm matter (Iwasawa \& Comastri 1998) from two AGN located in the two nuclei (Komossa et al. 2003). Above $10 \mathrm{keV}$, absorbed direct AGN emission has been observed with BeppoSAX and RXTE (Vignati et al. 1999; Ikebe et al. 2000). The question is to estimate the bolometric luminosity of the AGN from the observed direct and/or reflected AGN X-rays.

Spectral fits to the complete X-ray spectrum of NGC 6240 suggest an intrinsic (corrected for absorption) 2-10 keV luminosity of $2.2 \times 10^{43}$ to $2.7 \times 10^{44} \mathrm{erg} \mathrm{s}^{-1}$ (Vignati et al. 1999; Ikebe et al. 2000, corrected to the distance assumed in this paper). The main uncertainties involved in the analysis of the $2-10 \mathrm{keV}$ emission are the geometry of the X-ray reflector, and the potential effect of scattering by the absorbing matter. Similar uncertainties arise when extrapolating from the directly observed very hard (>10 keV) X-ray emission, due to the uncertain spectral index towards softer X-rays. In a next step, the
Table 3. Constraints on the contribution of starburst and AGN activity to the infrared luminosity of NGC 6240.

\begin{tabular}{lcc}
\hline \hline Constraint & Starburst & AGN \\
\hline Low excitation mid-IR lines & $50-100 \%$ & \\
PAH features & $>33 \%$ & \\
High excitation mid-IR lines & & $>24 \%$ \\
Mid-IR dust continuum & & $>13 \%$ \\
Hard X-ray emission & & $10-100 \%$ \\
\hline
\end{tabular}

$2-10 \mathrm{keV}$ luminosity has to be extrapolated to the bolometric luminosity. Assuming the mean radio-quiet quasar SED of Elvis et al. (1994, their Fig. 10$), L_{2-10 \mathrm{keV}} / L_{\mathrm{Bol}} \approx 0.09$, with significant scatter around this mean relation. In the regime of Seyferts, $L_{2-10 \mathrm{keV}} / L_{\mathrm{IR}}$ is typically around 0.1 for a major fraction of objects that are not absorbed in the 2-10 keV range, with a tail to smaller ratios (Risaliti et al. 2001). We have verified this by comparing observed $2-10 \mathrm{keV}$ fluxes of $z<0.1$ Seyfert 1s from George et al. (1998) and extinction-corrected 2-10 keV fluxes of Seyfert 2s from Bassani et al. (1999) with infrared fluxes. We have restricted the samples to objects where the absence of PAHs in ISOPHOT spectra suggests small star formation contribution, and excluded Compton thick objects. The mean $\log \left(L_{2-10 \mathrm{keV}} / L_{\mathrm{IR}}\right)$ for 18 objects (12 Sy1, $\left.6 \mathrm{Sy} 2\right)$ is -1.0 with a dispersion of 0.29 . Following our previous approach, we assume $L_{\mathrm{IR}} \approx 0.5 L_{\mathrm{Bol}}$ for the Seyferts.

In total, the AGN luminosity estimated from the X-ray spectroscopy is in the range $2 \times 10^{44}$ to $6 \times 10^{45} \mathrm{erg} \mathrm{s}^{-1}$, or between $10 \%$ and several times the luminosity of NGC 6240 , depending on the estimate of the X-ray luminosity and assumptions for the bolometric correction. The X-ray data suggest that the AGN plays a significant and possibly dominant role but cannot pin down the AGN luminosity accurately.

\subsection{Combining the constraints}

The five constraints on the contributions of star formation and AGN in NGC 6240 discussed in the previous subsections are summarized in Table 3. We assign highest weight to two constraints. The X-ray constraint suggests that the AGN is contributing a significant and possible dominant fraction of the luminosity, the latter situation being within the uncertainty of the estimate based on the observed $\mathrm{X}$-ray emission rather than just being allowed as an unseen obscured component. The second key constraint is the one on the starburst luminosity derived from the low excitation fine-structure lines, which is based on relatively straightforward analysis of starburst H II regions. It is difficult to envisage $\mathrm{H}$ II regions that are low excitation and still have a high ratio of ionizing luminosity to total luminosity, which would be needed to make the starburst contribution to the bolometric luminosity insignificant. We suggest that the most likely range of contributions to the luminosity of NGC 6240 is 50 to $75 \%$ starburst and 25 to $50 \%$ AGN. This estimate is based on direct comparison of mid-IR and X-ray observables to the bolometric luminosity. Reassuringly, the agreement with pure mid-IR diagnostics (Genzel et al. 1998; Laurent et al. 2000) is good. 


\section{Conclusions}

Infrared spectroscopy of NGC 6240 detects signatures of powerful star formation activity and of an obscured AGN. We have used these results in conjunction with X-ray spectroscopy to estimate the contributions to the total luminosity of starburst (50 to $75 \%$ ) and AGN activity (25 to $50 \%$ ). NGC 6240 is a luminous source of rotational emission of molecular hydrogen amounting to $0.3 \%$ of the bolometric luminosity, a similar amount is radiated in the [O I] $63 \mu \mathrm{m}$ line. Shocks due to the turbulent central velocity field and the superwind are likely to dominate these extraordinary levels of emission, and imply a mechanical luminosity of at least $10^{10} L_{\odot}$.

Acknowledgements. We acknowledge support by Verbundforschung (50 OR 9913 7), for the ISO spectrometer data center at MPE by DLR (50 QI 0202), and by the German - Israeli Foundation (grant I-0551186.07/97). We thank Olivier Laurent for access to his ISOCAM-CVF spectra, Matthias Tecza for providing $\mathrm{Br} \gamma$ data, and Stefanie Komossa for discussions. We are grateful to the referee for valuable suggestions.

\section{References}

Bassani, L., Daddina, M., Maiolino, R., et al. 1999, ApJS, 121, 473 Beswick, R. J., Pedlar, A., Mundell, C. G., \& Gallimore, J. F. 2001, MNRAS, 325, 121

Boulanger, F., Beichman, C., Désert, F. X., et al. 1988, ApJ, 332, 328

Capetti, A., Axon, D. J., \& Macchetto, F. D. 1997, ApJ, 487, 560

Clavel, J., Schulz, B., Altieri, B., et al. 2000, A\&A, 357, 839

Colbert, J. W., Malkan, M. A., Clegg, P. E., et al. 1999, ApJ, 511, 721

Dale, D., Helou, G., Contursi, A., Silberman, N. A., \& Kolhatkar, S. 2001, ApJ, 549, 215

de Graauw, Th., Haser, L. N., Beintema, D. A., et al. 1996, A\&A, 315, L49

Doyon, R., Wells, M., Wright, G. S., et al. 1994, ApJ, 437, L23

Draine, B. T., \& Woods, D. T. 1990, ApJ, 363, 464

Draine, B. T., \& Bertoldi, F. 1999, in The Universe as seen by ISO, ed. P. Cox, \& M. F. Kessler, ESA-SP427 (Noordwijk: ESA), 553

Egami, E. 1998, in The Central Regions of the Galaxy and Galaxies, ed. Y. Sofue (Dordrecht: Kluwer), IAU Symp., 184, 95

Egami, E. 1999, Ap\&SS, 266, 105

Elvis, M., Wilkes, B. J., McDowell, J. C., et al. 1994, ApJS, 95, 1

Fischer, J. 1999, in The universe as seen by ISO, ed. P. Cox, \& M. F. Kessler, ESA-SP427 (Nordwijk: ESA), 817

Förster-Schreiber, N. M., Genzel, R., Lutz, D., Kunze, D., \& Sternberg, A. 2001, ApJ, 552, 544

Förster-Schreiber, N. M., Sauvage, M., Charmandaris, V., et al. 2003, A\&A, 399, 833

Fried, J. W, \& Schulz, H. 1983, A\&A, 118, 166

Genzel, R., Lutz, D., Sturm, E., et al. 1998, ApJ, 498, 579

Genzel, R., Tacconi, L. J., Rigopoulou, D., Lutz, D., \& Tecza, M. 2001, ApJ, 563, 527

George, I. M., Turner, T. J., Netzer H., et al. 1998, ApJS, 114, 73

Goldader, J. D., Joseph, R. D., Doyon, R., \& Sanders, D. B. 1997, ApJS, 108, 449

Graham, J. R., Wright, G. S., \& Longmore, A. J. 1987 ApJ, 313, 847

Gry, C., et al. 2002, ISO Handbook, Vol. III, Version 2.0, http:// www. iso.vilspa.esa.es/manuals/HANDBOOK/lws_hb/

Heckman, T. M., Armus, L., \& Miley, G. K. 1990, ApJS, 74, 833

Hollenbach, D., \& McKee, C. F. 1989, ApJ, 342, 306

Ikebe, Y., Leighly, K., Tanaka, Y., et al. 2000, MNRAS, 316, 433

Iwasawa, K., \& Comastri, A. 1998, MNRAS, 297, 1219
Jones, A. P., Tielens, A. G. G. M., \& Hollenbach, D. M. 1996, ApJ, 469,740

Joseph, R. D., Wright, G. S., \& Wade, R. 1984, Nature, 311, 132

Kaufman, M. J., \& Neufeld, D. A. 1996, ApJ, 456, 611

Klaas, U., Haas, M., Heinrichsen, I., \& Schulz, B. 1997, A\&A, 325, L21

Klaas, U., Haas, M., Müller, S. A. H., et al. 2001, A\&A, 379, 823

Komossa, S., Schulz, H., \& Greiner, J. 1998, A\&A, 334, 110

Komossa, S., Burwitz, V., Hasinger G., et al. 2003, ApJ, 582, L15

Laurent, O., Mirabel, I. F., Charmandaris, V., et al. 2000, A\&A, 359, 887

Le Floc'h, E., Mirabel, I. F., Laurent, O., et al. 2001, A\&A, 367, 487

Lester, D. F., \& Gaffney, N. I. 1994, ApJ, 431, L13

Luhman, M. L., Satyapal, S., Fischer, J., et al. 1998, ApJ, 504, L11

Luhman, M. L., Satyapal, S., Fischer, J., et al. 2003, ApJ, 594, 758

Lutz, D., Kunze, D., Spoon, H. W. W., \& Thornley, M. D. 1998, A\&A, 333, L75

Lutz, D., Spoon, H. W. W., Rigopoulou, D., Moorwood, A. F. M., \& Genzel, R. 1998a, ApJ, 505, L103

Malhotra, S., Kaufman, M. J., Hollenbach, D., et al. 2001, ApJ, 561, 766

Maloney, P. R., Hollenbach, D., \& Tielens, A. G. G. M. 1996, ApJ, 466,561

Oliva, E., Lutz, D., Drapatz, S., \& Moorwood, A. F. M. 1999a, A\&A, 341, L75

Oliva, E., Moorwood, A. F. M., Drapatz, S., Lutz, D., \& Sturm, E. 1999b, A\&A, 343, 943

Poglitsch, A., Herrmann, F., Genzel, R., et al. 1996, ApJ, 462, L43

Rigopoulou, D., Spoon, H. W. W., Genzel, R., et al. 1999, ApJ, 118, 2625

Rigopoulou, D., Kunze, D., Lutz, D., Genzel, R., \& Moorwood, A. F. M. 2002, A\&A, 389, 374

Risaliti, G., Gilli, R., Maiolino, R., \& Salvati, M. 2000, A\&A, 357, 13

Rosenthal, D., Bertoldi, F., \& Drapatz, S. 2002, A\&A, 356, 705

Sanders, D. B., \& Mirabel, I. F. 1996, ARA\&A, 34, 749

Schaerer, D., \& Stasinska, G. 1999, A\&A, 345, L17

Schulz, H., Komossa, S., Berghöfer, Th. W., \& Boer, B. 1998, A\&A, 330,823

Solomon, P. M., Downes, D., Radford, S. E., \& Barrett, J. W. 1997, ApJ, 478, 144

Spinoglio, L., Malkan, M. A., Rush, B., Carrasco, L., \& Recillas-Cruz, E. 1995, ApJ, 453, 616

Spoon, H. W. W., Keane, J. V., Tielens, A. G. G. M., et al. 2002, A\&A 385,1022

Stacey, G. J., Geis, N., Genzel, R., et al. 1991 ApJ, 373, 423

Sternberg, A., \& Neufeld, D. 1999, ApJ, 516, 371

Sturm, E., Lutz, D., Tran, D., et al. 2000, A\&A, 358, 481

Sturm, E., Lutz, D., Verma, A., et al. 2002, A\&A, 393, 821

Sugai, H., Malkan, M. A., Ward, M. J., Davies, R. I., \& McLean, I. S. 1997, ApJ, 481, 186

Tacconi, L. J., Genzel, R., Tecza, M., et al. 1999, ApJ, 524, 732

Tacconi, L. J., Genzel, R., Lutz, D., et al. 2002, ApJ, 580, 73

Tecza, M., Genzel, R., Tacconi, L. J., et al. 2000, ApJ, 537, 178

Telesco, C. M., \& Decher, R. 1988, ApJ, 334, 573

Thornley, M. D., Förster Schreiber, N. M., \& Lutz, D., et al. 2000, ApJ, 539, 641

Timmermann, R., Bertoldi, F., Wright, C. M., et al. 1996, A\&A, 315, L281

Tran, Q. D., Lutz, D., Genzel, R., et al. 2001, ApJ, 552, 527

Turner, J., Kirby-Docken, K., \& Dalgarno, A. 1977, ApJS, 35, 281

van der Werf, P. P., Genzel, R., Krabbe, A., et al. 1993, ApJ, 405, 522

Verma, A., Lutz, D., Sturm, E., et al. 2003, A\&A, 403, 829

Verstraete, L., Puget, J. L., Falgarone, E., et al. 1996, A\&A, 315, L337

Vignati, P., Molendi, S., Matt G., et al. 1999, A\&A, 349, L57

Wolniewicz, L., Simbotin, I., \& Dalgarno, A. 1998, ApJS, 115, 293 